\title{
On the Preceding Paper "A Legendre Polynomial Integral" by James L. Blue
}

By Walter Gautschi

Abstract. The modified moments of the distribution $d \sigma(x)=x^{\alpha} \ln (1 / x) d x$ on $[0,1]$, with respect to the shifted Legendre polynomials, are explicitly evaluated.

The result in the theorem of Section 2 of [1] can be generalized as follows: Let

$$
\nu_{n}(\alpha)=\int_{0}^{1} x^{\alpha} \ln (1 / x) P_{n}^{*}(x) d x, \quad \alpha>-1, \quad n=0,1,2, \ldots,
$$

where $P_{n}^{*}(x)=P_{n}(2 x-1)$ is the shifted Legendre polynomial of degree $n$. Then

(1) $\nu_{n}(\alpha)=\left\{\begin{array}{l}(-1)^{n-m} \frac{m !^{2}(n-m-1) !}{(n+m+1) !}, \quad \alpha=m<n, m \geqslant 0 \text { an integer, } \\ \frac{1}{\alpha+1}\left\{\frac{1}{\alpha+1}+\sum_{k=1}^{n}\left(\frac{1}{\alpha+1+k}-\frac{1}{\alpha+1-k}\right)\right\} \prod_{k=1}^{n} \frac{\alpha+1-k}{\alpha+1+k}, \text { otherwise. }\end{array}\right.$

The result in [1] is the case $\alpha=0$ of (1). For the proof, we note that

$$
\nu_{n}(\alpha)=-2^{-(\alpha+1)} \int_{-1}^{1}(1+t)^{\alpha} \ln (1 / 2(1+t)) P_{n}(t) d t
$$

$$
=-2^{-(\alpha+1)} \lim _{\nu \rightarrow n}\left\{\int_{-1}^{1}(1+t)^{\alpha} \ln (1+t) P_{\nu}(t) d t-\ln 2 \cdot \int_{-1}^{1}(1+t)^{\alpha} P_{\nu}(t) d t\right\},
$$

where $P_{\nu}(t)$ is the Legendre function of degree $\nu$. It is well known [2, p. 316, Eq.

(15)] that

$$
\int_{-1}^{1}(1+t)^{\alpha} P_{\nu}(t) d t=\frac{2^{\alpha+1} \Gamma^{2}(\alpha+1)}{\Gamma(\alpha+\nu+2) \Gamma(\alpha+1-\nu)}, \quad \alpha>-1 .
$$

Differentiating (3) with respect to $\alpha$ gives

$$
\begin{aligned}
& \int_{-1}^{1}(1+t)^{\alpha} \ln (1+t) P_{\nu}(t) d t \\
& \quad=\frac{2^{\alpha+1} \Gamma^{2}(\alpha+1)}{\Gamma(\alpha+\nu+2) \Gamma(\alpha+1-\nu)}\{\ln 2+2 \psi(\alpha+1)-\psi(\alpha+\nu+2)-\psi(\alpha+1-\nu)\}
\end{aligned}
$$

with $\psi(x)=\Gamma^{\prime}(x) / \Gamma(x)$ the logarithmic derivative of the gamma function. The assertion (1) now follows by inserting (3) and (4) in (2) and by using the recurrence relations $\Gamma(x+1)=x \Gamma(x), \psi(x+1)=\psi(x)+1 / x$, together with the fact that for any integer

Received May 31, 1978.

AMS (MOS) subject classifications (1970). Primary $33 \mathrm{~A} 65$.

(C) 1979 American Mathematical Society 0025-5718/79/0000-0069/\$01.50 
$r \geqslant 0$,

$$
\frac{\psi(-r+\epsilon)}{\Gamma(-r+\epsilon)} \rightarrow(-1)^{r-1} r ! \quad \text { as } \epsilon \rightarrow 0 .
$$

The method of proof also allows the evaluation of integrals of the form

$$
\nu_{n, k}(\alpha)=\int_{0}^{1} x^{\alpha}[\ln (1 / x)]^{k} P_{n}^{*}(x) d x,
$$

by repeatedly differentiating (4) with respect to $\alpha$.

Department of Computer Sciences

Purdue University

West Lafayette, Indiana 47907

1. J. L. BLUE, “A Legendre polynomial integral," Math. Comp., v. 33, 1979, pp. 739-741.

2. A. ERDÉLYI (Ed.), Tables of Integral Transforms, Vol. II, McGraw-Hill, Now York, 1954. 